\title{
Identification of potential sources of airborne Olea pollen in the Southwest Iberian Peninsula
}

\author{
S. Fernández-Rodríguez (1), C. Ambelas Skjøth (2,3), R. Tormo-Molina (1), R. Brandao (4), E. Caeiro (4), I. \\ Silva-Palacios (5), Á. Gonzalo-Garijo (6), and M. Smith (7) \\ (1) Department of Plant Biology, Ecology and Earth Sciences, Faculty of Science, University of Extremadura, Science \\ Faculty, Avda. Elvas s/n, 06071 Badajoz, Spain, (2) Department of Earth and Ecosystem Sciences Division of Physical \\ Geography and Ecosystems Analysis, Lund University, Sweden, (3) Faculty of Science and Technology, Department for \\ Environmental Science, Aarhus University, Denmark, (4) Laboratory of Palynology and Aerobiology, Patio ICAAM Matos \\ Rosa, Mitra. Évora. Portugal, (5) Department of Applied Physics, Engineering Agricultural School, Uiversity of of \\ Extremadura. Badajoz. Spain, (6) Section of Allergology, Hospital Infanta Cristina. Badajoz, Spain, (7) Aerobiology and \\ Pollen Information Research Unit, Department of Oto-Rhino-Laryngology, Medical University of Vienna, Vienna, Austria
}

This study aims to determine the potential origin of Olea pollen recorded in Badajoz in the Southwest of the Iberian Peninsula during 2009-2011. This was achieved using a combination of daily average and diurnal (hourly) airborne Olea pollen counts recorded at Badajoz (southwestern Spain) and Évora (southeastern Portugal), an inventory of olive groves in the studied area and air mass trajectory calculations computed using the HYSPLIT model. Examining olive pollen episodes at Badajoz that had distinctly different diurnal cycles in olive pollen in relation to the mean, allowed us to identify three different scenarios where olive pollen can be transported to the city from either distant or nearby sources. Back trajectory analysis showed that olive pollen can be transported to Badajoz from the West on prevailing winds, either directly or on slow moving air masses, and from high densities of olive groves situated to the Southeast (e.g. Andalucía). Regional scale transport of olive pollen can result in increased nighttime concentrations of this important aeroallergen. This could be particularly important in Mediterranean countries where people can be outdoors during this time due to climate and lifestyle. Such studies are valuable for allergy sufferers and health care professionals because the information can be incorporated into forecasts, the outputs of which are used for avoiding exposure to aeroallergens and planning medication. The results of studies of this nature can also be used for examining gene flow in this important agricultural crop. 